\title{
Implementing e-Upchaar: Hospital Management Information System for public health facilities in Haryana
}

\author{
Sonia Trikha ${ }^{1}$, Bhavnish Arora ${ }^{2}$, Meenakshi Sharma ${ }^{3}$, Parneeta Thakur $^{4}$
}

${ }^{1}$ MD, Executive Director, State Health Systems Resource Centre, Haryana, India

${ }^{2}$ PGDHHM, Deputy Director, State Health Systems Resource Centre, Haryana, India

${ }^{3} \mathrm{PhD}$ in Public Health, PGIMER, Chandigarh, India

${ }^{4}$ MBA in Hospital Management, Domain expert and Business analyst, State Health Systems Resource Centre, Haryana, India

\section{Type of article: Original}

\begin{abstract}
Background and objective: There is a paucity of reliable hospital data and available information for planning and management of health services in India. Hence, the Government of Haryana launched e-Upchaar, a Hospital Management Information System (HMIS) in the public health facilities of the state. A study was conducted to give insight into the design, development and implementation of HMIS for public health facilities, capable of meeting futuristic health demands of Haryana state and the introduction of real-time reporting, data analytics for evidence-based rational decision making.

Methods: A descriptive study (August-October 2019) was conducted in the state of Haryana, India on implementation of HMIS (2015-2019). Desk reviews and interviews were conducted and the data from back-end of the application was explored. Integration of an external application by the Government of India's Ministry of Health initiative 'Mera Aspataal' with e-Upchaar was also reviewed. The data extracted from the database was saved in Excel 2010 for descriptive analysis.

Results: A unique health ID (UHID) was created for every patient registered in e-Upchaar. Patients (80 lakhs) and EMRs (70 lakhs) created untill 2019. Registration time (1 minute), follow up visits (15 sec), billing (2 minutes) and diagnostic reports' availability on the same day. This resulted in a significant reduction in operational costs; savings due to non-printing of X-ray films in 22 facilities amounted to 13 million INR.

Conclusion: e-Upchaar is playing a pivotal role towards achieving the quality standards for effective health care delivery in Haryana. It has improved transparency and integrity in hospital operations through accurate inventory management; and brought improvement in patients' experience.

Keywords: Hospital Management Information System (HMIS), Information System, Health Information System, e-Upchaar, Haryana, India
\end{abstract}

\section{Abbreviations / Acronyms:}

DCH: District Civil Hospital; DC: Data Centre; FRU: First Referral Unit; GMC: Government Medical College; HMIS: Hospital Management Information System; ICD: International Statistical Classification of Diseases and Related Health Problems; ICD-10: the 10th revision of ICD; INR: Indian Rupee; ISMO: Information Security Management Office; LAN: Local Area Network; MPLS: Multi-protocol label switching; PHC: Primary Health Care; RFP: Request For Proposals; SDH: Sub-divisional Hospital; UHG: UnitedHealth Group; UHID: unique health ID; UPHC: Urban Primary Health Centre; WAN: Wide Area Network.

\section{Corresponding author:}

Dr. Sonia Trikha. State Health Systems Resource Centre, Haryana, India. Tel: +91.1722590629 and +91.9560795333, E-mail: edhshrc@gmail.com

Received: December 10, 2019, Accepted: February 26, 2020, Published: June 2020

iThenticate screening: Februatu 26, 2020, English editing: May 29, 2020, Quality control: June 03, 2020

This article has been reviewed / commented by three experts

(C) 2020 The Authors. This is an open access article under the terms of the Creative Commons Attribution-NonCommercialNoDerivs License, which permits use and distribution in any medium, provided the original work is properly cited, the use is non-commercial and no modifications or adaptations are made. 


\section{Introduction}

The health information system is one of the six building blocks that describe health systems formulated by the World Health Organization for health system strengthening (1). Globally, there has been growing emphasis on the necessity for Hospital Management Information Systems (HMIS). The functions of HMIS include storing patients' information including but not limited to recording diagnosis of the patients, generating Electronic Medical Records, generating bills, managing HR, equipment maintenance, ensuring availability of drugs and diagnostics (2). Literature has shown the benefits of implementing HMIS e.g. reducing medical documentation errors, decreasing expenditure on manual and paper costs, preventing billing-related errors etc. (3). Due to increase in demand of health care services, it is hard to perform documentation manually in hospitals. Therefore, emphasis has been laid on the shift from manual to electronic system to automate all the necessary operations in a hospital. The developed world paid thoughtful attention to HMIS, and Health Information Technology was placed at the heart of the Federal Government Program in America in 2004 (4). In India, the 12th draft of the 5-year plan mentions the need to strengthen HMIS across the country (3). Ministry of Electronics and Information Technology, Government of India released e-Sushrut C-DAC's HMIS in 2014 towards adapting technology to improve healthcare (5). HMIS known as e-Upchaar (6) has been successfully implemented in public health institutions of Haryana state in India since 2015 , but it is in nascent stage in the rest of the country. Hence this study is aimed to give insight into the design, development and implementation of HMIS for public health facilities capable of meeting futuristic health demands of Haryana state and the introduction of real-time reporting, and data analytics for evidence-based rational decision making.

\section{Material and Methods}

A descriptive study (August- October 2019) was conducted in the state of Haryana, India on the implementation of IT-based HMIS (2015-2019). Desk reviews and interviews of the key stakeholders viz. Deputy Director (Quality \& Healthcare technology) (1), Domain (HMIS) expert (1), Principal Medical officers (5), Radiologists (4) and other officials responsible for HMIS were conducted to explore the design, processes and development of the HMIS in Haryana state and to compare the manual system with computerized HMIS. The data from the back-end of the application was explored to understand real-time reporting and data analytics. Integration of the application 'Mera Aspataal (7)' (Ministry of Health, Government of India initiative to capture patient feedback on hospital services) with e-Upchaar was also reviewed. The data extracted from the database was saved in Excel 2010 to conduct the descriptive analysis. Permission for the study was obtained from the concerned authorities of the State Health System Resource Centre, Haryana (HSHRC); the main implementing agency of e-Upchaar.

\section{Results}

\subsection{Design, development and implementation}

The envisioning for information and communication technology in Haryana for health care delivery was initiated (2013-2014) with input from the National Institute of Smart Governance. It was proposed that e-Upchaar would be a one-core, automated, scalable and integrated software application, deployed centrally at Data Centre (DC) in the state of Haryana, having a Web Portal interface accessible through DC and Intranet core. Request For Proposals (RFP) was invited from the established system implementers known for their expertise in implementing HMIS application, and the contract was awarded to M/s UHG presently known as M/s OGS, after stringent evaluation based on quality. The technology reference architecture (Figure 1) explained the integration of the different hospital users and synchronization of all the activities.

The committee comprising the Director General of Health Services, Director General Medical Education and Research, Director- AYUSH and Executive Director- HSHRC designated the software package as 'e-Upchaar'. After a memorandum of understanding was signed in 2014, the first phase of the system which included 18 modules was installed in four public health care facilities of Haryana in 2015. Pilot testing was carried out at four sites (one PHC, one CHC, one District Civil Hospital (DCH) and one Government Medical College (GMC) (Table 1). Before implementation of the application; the standard software testing life cycle protocol was followed, user acceptance testing was conducted and customization done accordingly. Finally, the security testing was done by the Information Security Management Office (ISMO), Haryana to ensure that the data contained in the application is secure and conforms to the security standards prevalent in the industry. The application was developed in Java software (frontend) and MySQL 5.7 (back-end). Figure 2 shows the process flowchart that briefly elaborates the HMIS decision system for supporting all levels of clinical or hospital operations. 


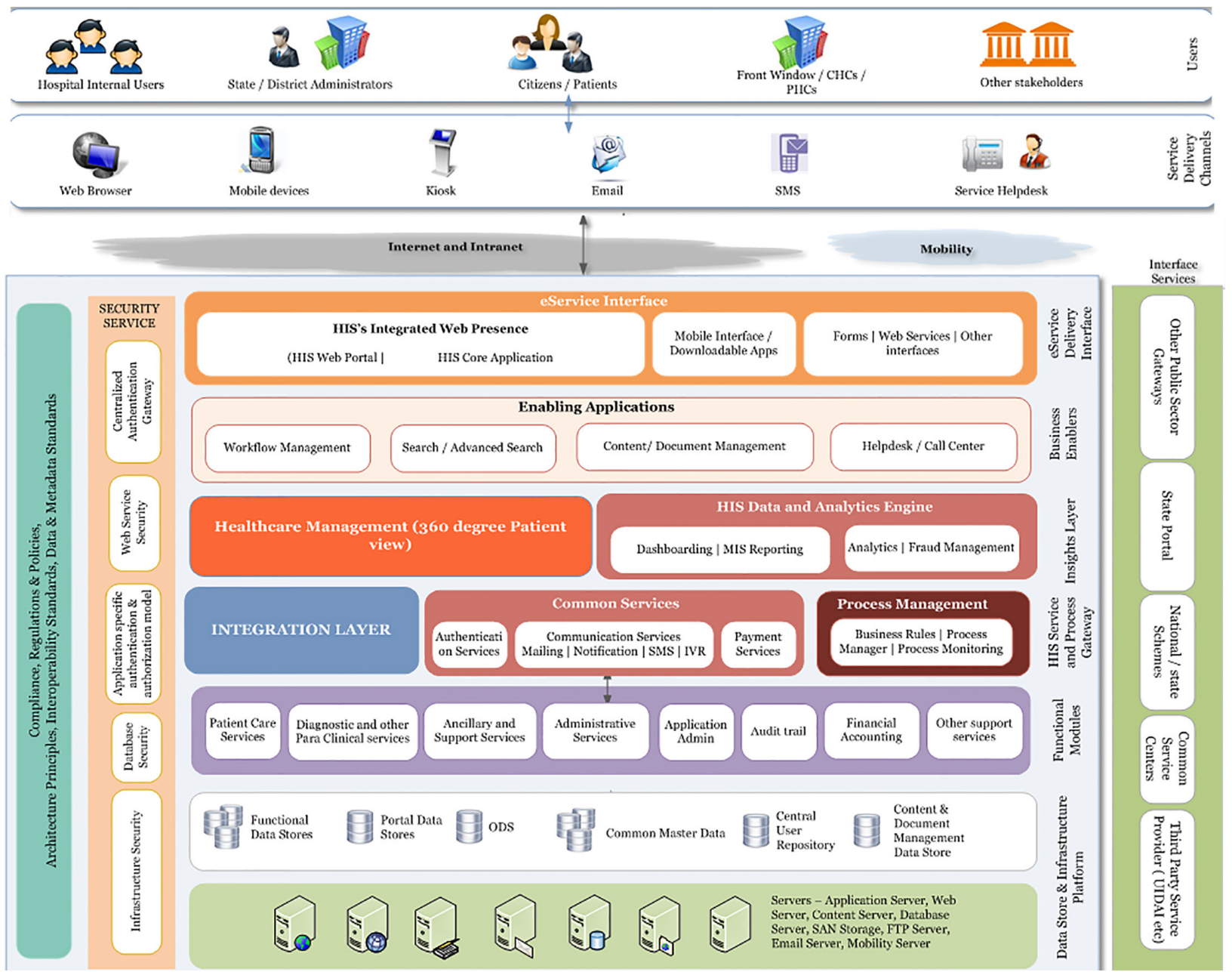

Figure 1. Technology reference architecture of the HMIS

Table 1. Evolution of HMIS in Public Health facilities of Haryana

\begin{tabular}{|l|l|l|l|}
\hline Features & Phase 1 (2015-2016) N & Phase 2 (2016-2017) N & Phase 3 (2018-2019) N \\
\hline No. of health care facilities & $\begin{array}{l}4 \text { [PHC (1), CHC (1), } \\
\text { DCH (1), MC (1)] }\end{array}$ & $\begin{array}{l}\text { 18 [DCH (16), MC (1), } \\
\text { AYUSH MC (1)] }\end{array}$ & $\begin{array}{l}\text { 34 [DCH (5), MC (1), } \\
\text { SDH (12) } \\
\text { FRU (2), CHC (4), } \\
\text { PHC (6) } \\
\text { UPHC (3)*] }\end{array}$ \\
\hline No. of modules & 17 & 19 & 20 \\
\hline $\begin{array}{l}\text { Users trained (doctors, staff nurses \& other } \\
\text { health care providers) }\end{array}$ & 1500 & 3200 & 6500 \\
\hline Key performance indicators & - & - & 21 \\
\hline Supervisory visits & 11 & 22 & 36 \\
\hline
\end{tabular}

*PHC: Primary Health Centre, DCH: District Civil Hospital, MC: Medical College, SDH: Sub-divisional Hospital, FRU: First Referral Unit, UPHC: Urban Primary Health Centre

In 2016, the second phase was implemented across 18 sites DCH (16), MC (1), AYUSHMC (1) and billing module was introduced in addition to existing 18 modules viz. Registration, OPD, Laboratory, Radiology, IPD, Pharmacy, Labor room, Emergency, OT, Blood bank, Birth and death registration, Central sterile and supply department, Equipment management, Store and sub store management, MRD, Maintenance and Purchase. In Phase-3 (2019), HMIS was implemented in remaining District Civil Hospitals (5), MC (1) and Sub-divisional Hospitals (SDH) (11). The Human Resource Management System (HRMS) module including duty roster management, training and leave management was introduced to augment the system. Twenty one Key Performance Indicators (KPIs) were also 
applied to measure the performance of various health facilities of Haryana (Box 1). Tracking these measurable KPIs facilitated the users to visualize areas where services can be optimized, and highlight problems that can be resolved with desired interventions. The e-Upchaar is also compliant with existing standards viz.; HL7 interoperability standards, EHR2016 and DICOM3.0 compliant, SNOMED-CT and ICD10 classification to ensure futuristic health demands.

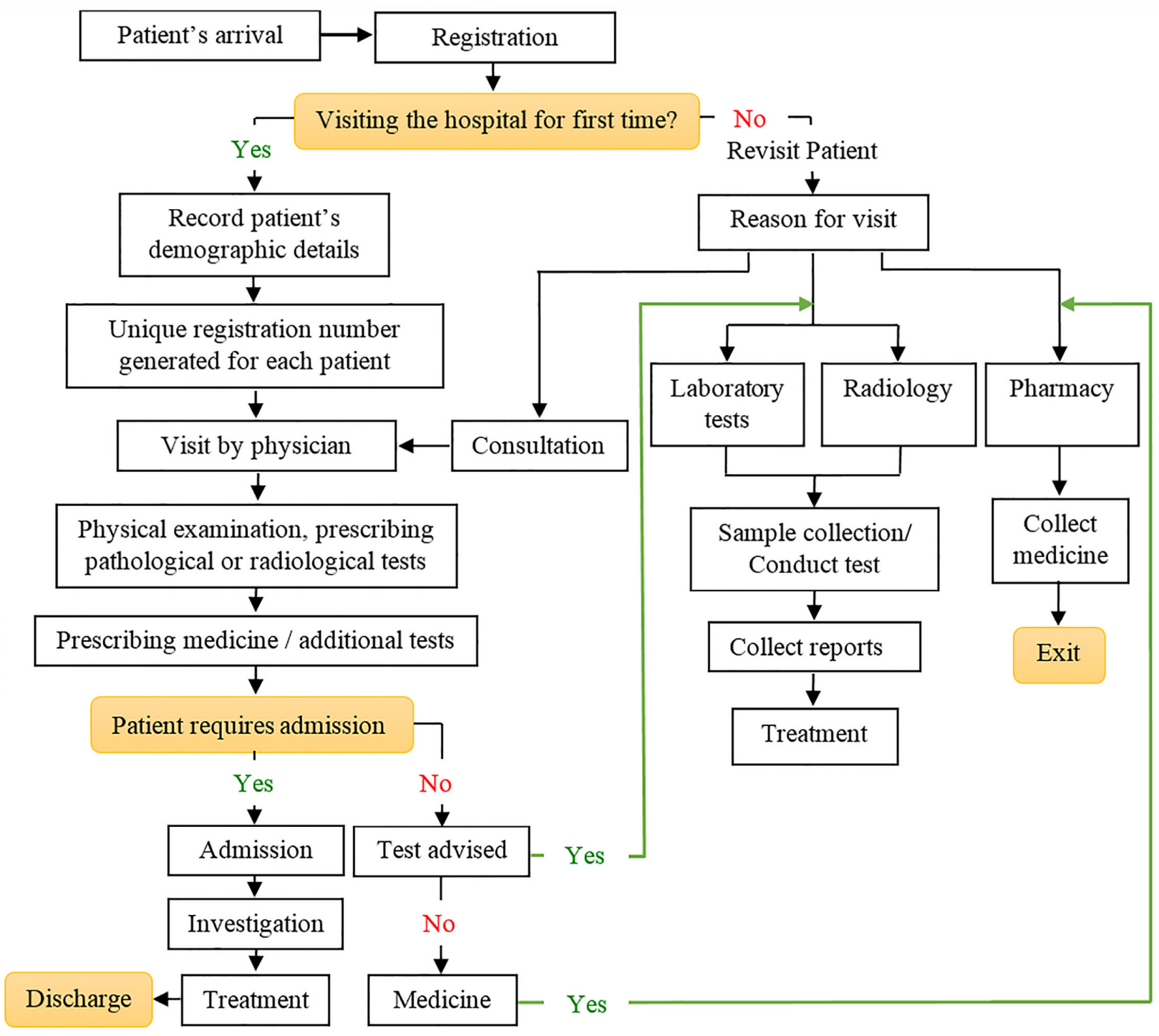

Figure 2. Flowchart of the HMIS processes

Box1. Key Performance indicators of HMIS in the public health facilities of Haryana

\begin{tabular}{|l|l|}
\hline 1. Outpatient department waiting time & 12. Neonatal mortality \\
2. Laboratory investigation turnaround time & 13. OT utilization \\
3. Out of range results & 14. OT performance \\
4. Radiology investigations waiting and turnaround time & 15. Re -exploration \\
5. Pharmacy average turnaround time & 16. Blood bank \\
6. Bed occupancy & 17. Stock out of essential drugs \\
7. Readmission & 18. Near-Expiry drugs and consumables \\
8. Discharge turnaround time & 19. Non-functional Equipment analysis \\
9. Discharge without discharge summary & 20. Number of Inpatient deaths \\
10. Institutional births & 21. Referral analysis \\
11. Normal Vs Caesarean Vs Assisted Deliveries & \\
\hline
\end{tabular}




\subsection{Real-time reporting, data analytics for evidence-based rational decision making}

The user interface of the HMIS is menu-driven and easy to use. User guidance is available through helpful content inside the tooltips. All users are provided training before the implementation which is refreshed on a regular basis (Table 1). Individual login is provided to the users (doctors, staff nurses, pharmacists, lab technicians, data entry operators etc.). The application is available on a closed MPLS (Multi-protocol label switching) network and can be accessed at the facility only. Recording and reporting is captured, the moment it occurs at the sites. Essentially, eUpchaar allows analytics of data that are captured at various nodes. Improvement in operational efficiency of hospital and saving patients precious time is one of the major advantages of e-Upchaar. Table 2 shows utilization of service indicators e.g. number of new patients registered/electronic medical record (EMR) generated $(53,21,378)$, number of drugs dispensed through pharmacy module etc. A unique health ID (UHID) is provided to each patient registered in e-Upchaar. This helps in maintenance and retrieval of EMR when the patient revisits for follow up. EMR captures a range of data; including demographics, medical history, medication and allergies, laboratory test results, radiology images (Picture Archiving and Communication System), vital signs and the treatment during the course of hospital stay including any medical/surgical procedures, and subsequent visits.

Table 2. Service delivery indicators for HMIS in public health facilities in Haryana since 2015

\begin{tabular}{|l|l|l|l|l|}
\hline Data Type & 2015 & 2016 & 2017 & 2018 \\
& $\mathrm{~N}$ & $\mathrm{~N}$ & $\mathrm{~N}$ & $\mathrm{~N}$ \\
\hline Registration for new patients & $4,74,609$ & $39,79,613$ & $45,53,795$ & $53,21,378$ \\
\hline Registration for revisit patients & $3,24,036$ & $26,75,043$ & $39,13,876$ & $63,36,466$ \\
\hline IPD registrations & 23,656 & $29,05,58$ & $40,88,02$ & $50,22,46$ \\
\hline Doctors consultation in general OPDs & $7,78,467$ & $64,70,686$ & $83,25,476$ & $11,52,3722$ \\
\hline X- ray usage & 44,619 & $2,20,837$ & $3,42,601$ & $6,46,723$ \\
\hline Lab investigation requisition slip & $1,80,725$ & $6,48,790$ & $9,33,519$ & $1,80,1983$ \\
\hline Lab reports & $1,80,725$ & $6,48,288$ & $9,33,040$ & $1,79,8241$ \\
\hline Surgeries conducted in OTs & 80 & 2769 & 10,771 & 40,555 \\
\hline Drugs dispensed in the pharmacy & $92,43,447$ & $10,5162,430$ & $13,5019,398$ & $43,5623,471$ \\
\hline
\end{tabular}

The doctors are able to check the real-time availability of essential drugs in the hospital. This saves the patients' as well as the doctor's time and effort in case the prescribed drug is not available in the pharmacy and the prescription needs to be changed. The application alerts the pharmacy and stores with regard to the stock position of the drugs and consumables. Figure 3 depicts the bed occupancy under IPD-KPI dashboard of e-Upchaar; which is gathered, analyzed and packaged to view results on a routine basis. This helps in allocation of resources; researching the causes and effects of bed shortages. Introduction of ICD10 classification in HMIS has improved clinical efficiency (Figure 4) as the data generated by the system acts as a foundation for the identification of health trends, disease burden, statistics, and is instrumental in the planning for the ensuing year. For the convenience of doctors, the commonly used diagnosis terminology is mapped to facilitate users.

(-)-Uphaar Project objectives - KPls

- \& Dr Parneeta -

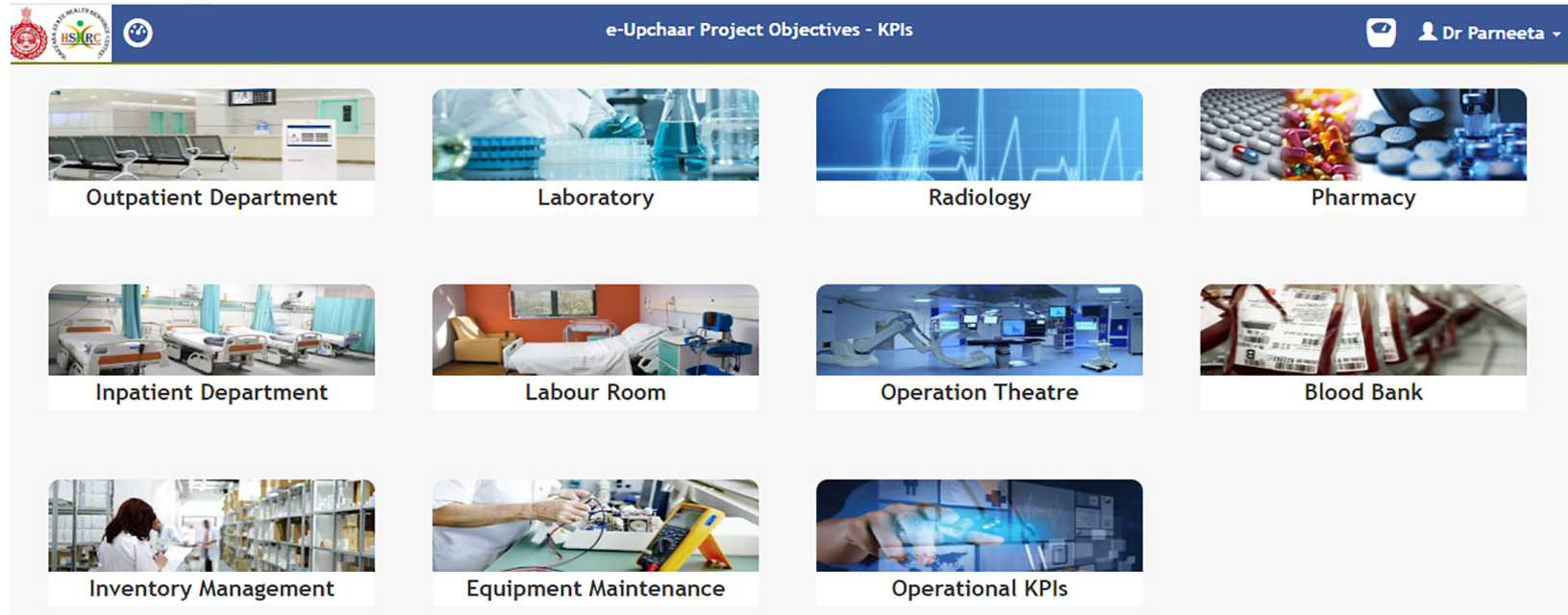

Figure 3. Bed occupancy (1.1.2019 - 30.09.2019) as shown on the KPI-IPD dashboard of HMIS system of public health facilities of Haryana 


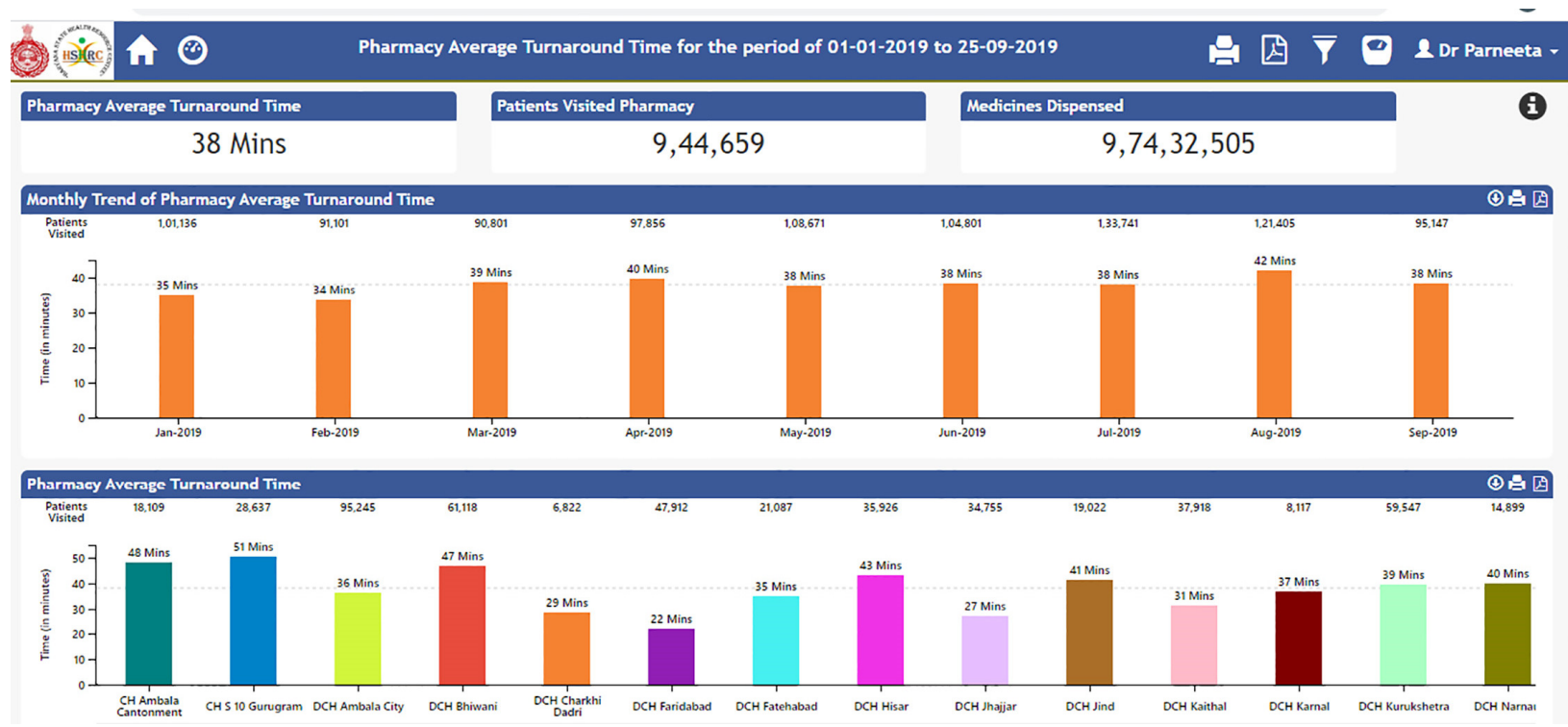

Figure 4. ICD10 classification in HMIS for improving clinical efficiency

In 2016, based on the billing module, a discrepancy regarding charges for registration, e.g. medico-legal charges at the various health facilities was observed. Based on this, standardization of charges was done by a gazette notification (8) issued in 2017; accordingly the billing module was successfully implemented. With the integration of the radiological equipment, digital transmission of images has been achieved. This has eliminated the need to manually file, retrieve, or transport printed copies of radiographs. It contributed to a significant reduction in operational costs e.g.,82\% (307907) of the X-ray films were digitalized and resulted in saving approximately 13 million INR from 22 facilities (Jan-Jun,2019) (Figures 5, 6). Now, the images are printed on-demand and only if cross consultations are required.

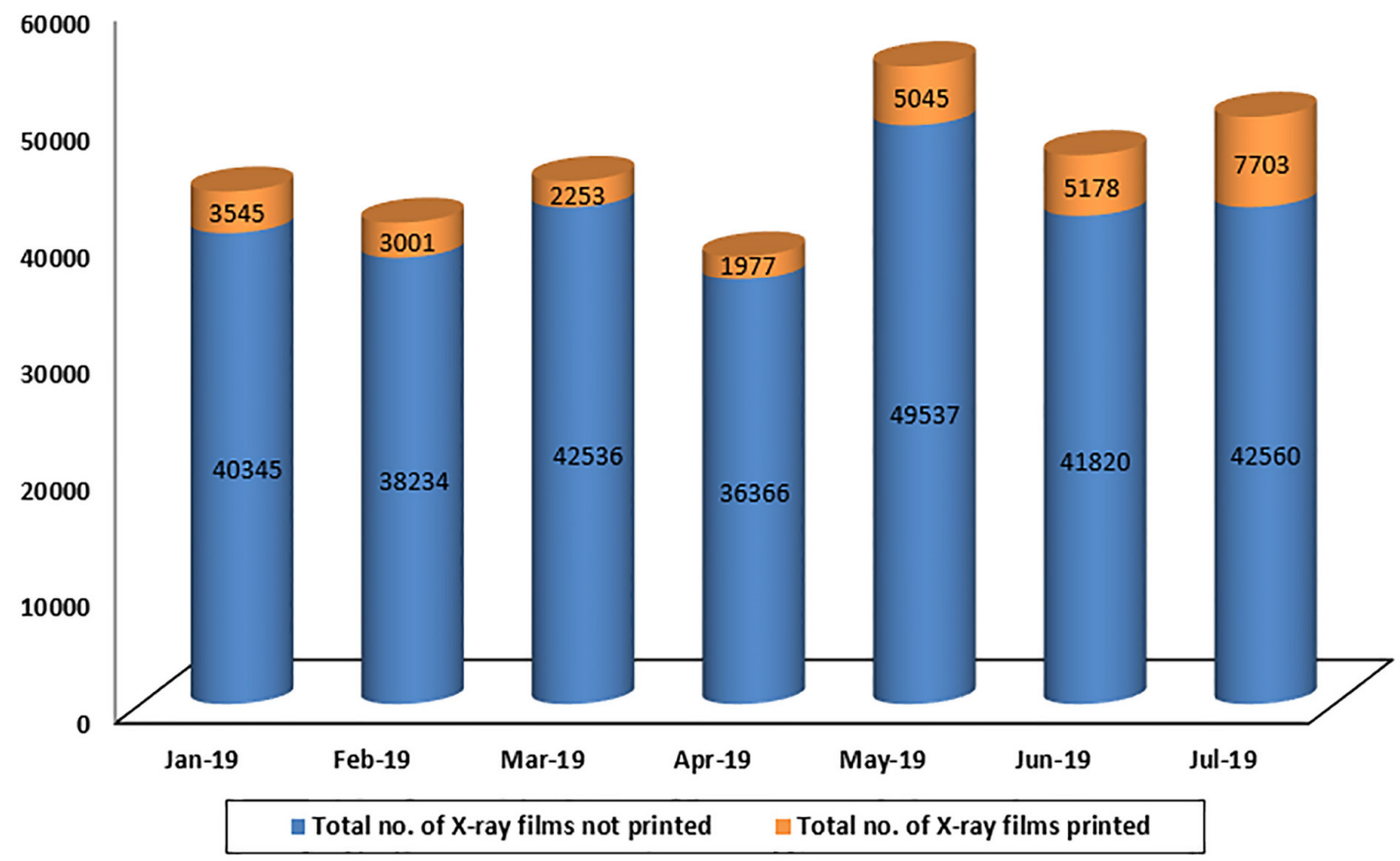

Figure 5. Number of the X-ray films printed during 2019 


\section{Total savings (in lakhs)}

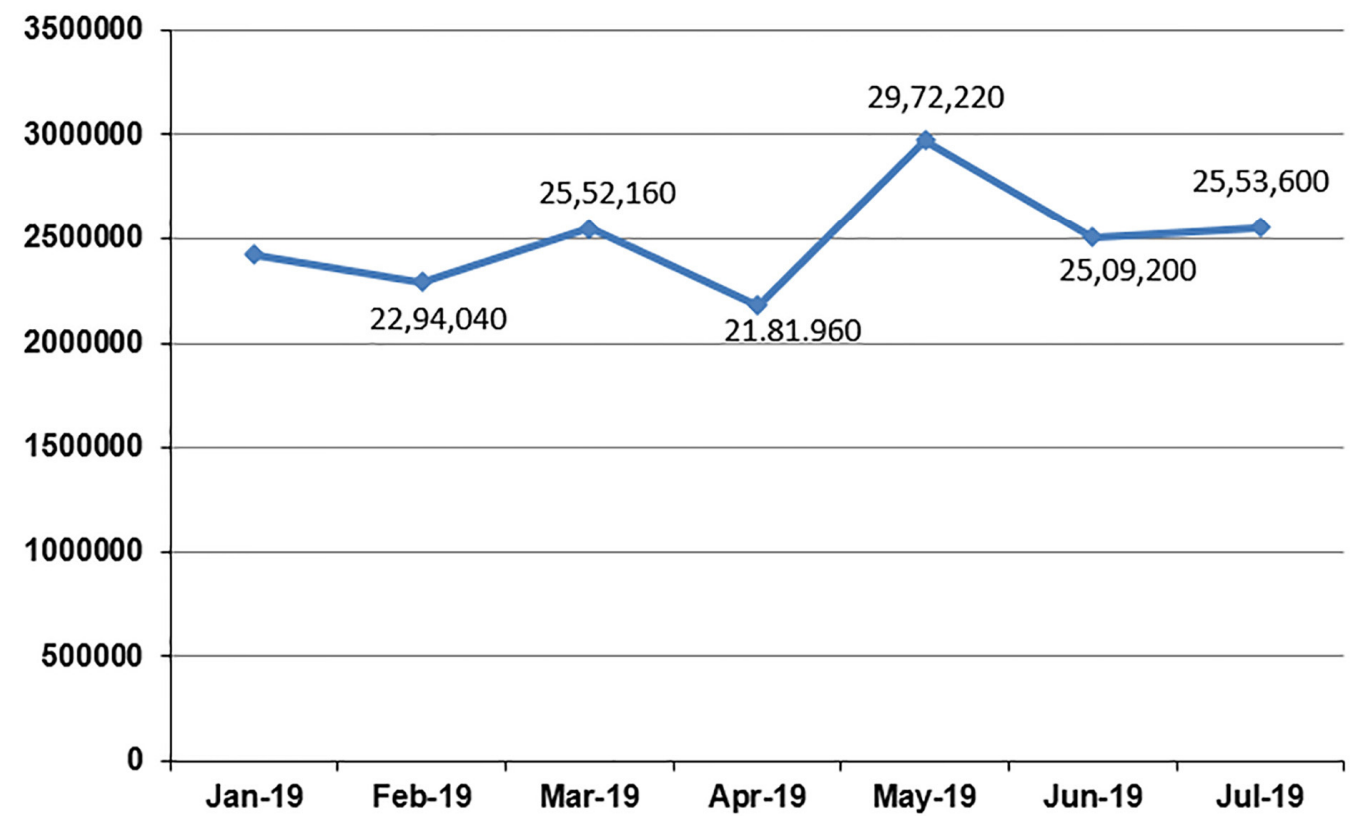

Figure 6. Savings from non-printing of X-ray films due to integration in HMIS Cost effectiveness

The integration of e-Upchaar with 'Mera Aspataal'(7) application empowers the patients to share feedback about services provided in the facility and leads to improvement in the delivery. One of the main advantages of e-Upchaar is that it has saved a lot of precious time. Health care workers took more than double the time to update their records manually as compared to e-Upchaar (average time taken for billing was 2-4 hours as compared to 2-3 minutes in eUpchaar) (Table 3).

Table 3. Comparison on the manual versus e-Upchaar HMIS based on stakeholder's perspectives

\begin{tabular}{|c|c|c|}
\hline Services/Facilities & Manual system & e-Upchaar* \\
\hline Patient Registration & $\begin{array}{l}4-5 \text { minutes per new patient once the patient } \\
\text { reaches the counter }\end{array}$ & 1 minute per new patient \\
\hline $\begin{array}{l}\text { Follow Up visit with } \\
\text { UHID }\end{array}$ & $\begin{array}{l}\text { 3-4 minutes per patient [In practice, a new } \\
\text { card is issued to revisiting patients] }\end{array}$ & 15 seconds per patient \\
\hline $\begin{array}{l}\text { Billing \& Cash } \\
\text { Collection }\end{array}$ & $2-4$ hours per patient & 2-3 minutes \\
\hline $\begin{array}{l}\text { Diagnostic report } \\
\text { availability }\end{array}$ & 1 - 2 days & Same day in most cases \\
\hline Inventory Control & Poor \& lengthy system of management & $\begin{array}{l}\text { Reduction in wastage with alerts for near } \\
\text { expiry and expired stock }\end{array}$ \\
\hline Blood Bank & $\begin{array}{l}\text { - No track of bloodstock, information on } \\
\text { available blood groups etc. } \\
\text { - Manual tracking of blood bag expiry } \\
\text { date } \\
\text { - Manual Tracking of blood collected } \\
\text { during blood camps }\end{array}$ & $\begin{array}{l}\text { - } \text { Increase in blood utilization } \\
\text { - } \quad \text { Centralized Blood Inventory } \\
\text { Information } \\
\text { - } \quad \text { Time and cost-saving makes blood } \\
\text { banking productive }\end{array}$ \\
\hline $\begin{array}{l}\text { Care planning by } \\
\text { physicians }\end{array}$ & $\begin{array}{l}\text { Care planning is event-based and time } \\
\text { consuming }\end{array}$ & $\begin{array}{l}\text { EMR of a patient helps physician in better } \\
\text { care planning and monitoring }\end{array}$ \\
\hline
\end{tabular}

\section{Discussion}

* Based on LAN/WAN Service level agreement monitoring

Literature has suggested the necessity for enhancing information systems and the role of information technology in health care (9-11); hence, HMIS is essential for an effective health system in any country (12). In India, the implementation of HMIS is in a nascent stage and therefore, there is often a lack of useful quality data and 
inefficient utilization of resources (13). Downey et al. emphasized that the lack of consistent and quality data (14) for planning and management of health services is a significant weakness of the health systems in our country (13). To tackle this problem, the Government of Haryana launched its flagship project to implement e-Upchaar (6) in the public health facilities of the state. The current study was conducted to understand the design, development, and implementation of HMIS in Haryana. Our experience at Haryana showed that the application of HMIS is feasible and has several advantages. The current study depicted that e-Upchaar has attained most of what it set out to accomplish in terms of improving the quality of data and timeliness at the level of generation. The e-Upchaar project has facilitated the implementation of a reliable system for service delivery, planning, monitoring, and supervision. The current study emphasized the importance of computerized patient records and the integrity of information to provide better services. This is consistent with the findings of other studies that have reported the role of information technology in strengthening the health systems of developing countries $(9,13)$.

One of the advantages was in time saved by the e-Upchaar, whether it was a registration of new patients, follow up, billing, or checking real-time availability of essential drugs. The current study showed that registration of a new patient took four times lesser time as compared to manual registration. This is consistent with findings by other studies, which showed that the average time taken for registration of new patients in HMIS was low $(3,13)$.

Our study reported that data generated by e-Upchaar plays a significant role in the identification of health trends, disease burden, and statistics. Kalankesh et al. (15) depicted the trends, patterns in morbidity, and mortality through data from HIS for an informed decision in health care management. These trends explain the dynamics of diseases in a given population and, consequently, the health care demands of the community.

The present study also showed a significant reduction in operational costs, e.g., by non-printing of X-ray films. The substantial cost and workforce savings are highly important in resource constraint settings of developing countries. Moreover, the digitalization of X-rays is expected to make the health system more efficient and further improvement in communication among physicians. Arora et al. (16) also showed that picture archiving and communication systems are important tools for digitizing, archiving and transmission of the image in a cost-effective way and reported a saving of 0.7 million per month in ICUs for radiology film.

\section{Study strengths and limitation}

Despite the availability of literature, the exact knowledge-based HMIS is not fully implemented in India. The current study provides glimpses of a success story of the implementation of HMIS in public health facilities of India and presents the development stages of HMIS. A limitation of this study could be that the perspectives of patients were not taken, and only health care professionals were included as respondents. Therefore, future studies should involve patients to provide a holistic view of the characteristics of HMIS. Though interviews of the key stakeholders were carried out in a neutral unbiased manner, the possibility of interviewer bias could not be ruled out. Another limitation could be generalizing its findings to the whole of country, due to the organizational and behavioral challenges in various settings.

\section{Conclusions}

The e-Upchaar application has improved transparency and integrity in hospital operations through accurate inventory management, and transparent reporting and has brought an improvement in patients' experience through efficient hospital queue management, medicine dispensing, etc. Hence, this success story needs to be replicated with a focus on change management in the rest of the country.

\section{Acknowledgments:}

We are thankful to Government of Haryana, India and State Institute of Health \& Family Welfare, Haryana, India for providing support for conducting the study.

\section{Conflict of Interest:}

There is no conflict of interest to be declared.

\section{Authors' contributions:}

Conception or design of the work (ST, BA), Acquisition of data (ST, BA, PT), Analysis or interpretation of data (All authors), Drafting the manuscript (ST, BA, MS), Revising the manuscript (All authors), Accountable for all aspects of the work (All authors). 


\section{References:}

1) Manyazewal T. Using the World Health Organization health system building blocks through survey of healthcare professionals to determine the performance of public healthcare facilities. Arch Public Health. 2017; 75:50. DOI: 10.1186/s13690-017-0221-9 PMid: 29075485 PMCid: PMC5651704

2) Abdulla Mohammed,Al-Mejibli Intisar SK. An Investigation Study of Hospital Management Information System. Int J Adv Res Comput Commun. 2017 [cited 2019 Sep 25];6(1). DOI: 10.17148/IJARCCE.2017.6184

3) Mukherjee K, Karuppiah P, Babu A. Economic evaluation of hospital management information systems in Tamil Nadu, India. Int J Med Public Heal. 2014;4(3). Available from: https://pdfs.semanticscholar.org/a6dc/44ef8ba71c4e9c8631dd869a57e2f008b5ad.pdf

4) Moghaddasi H, Mohammadpour A, Bouraghi H, Azizi A, Mazaherilaghab H. Hospital Information Systems: The status and approaches in selected countries of the Middle East. Electron physician. 2018 May;10(5):6829-35. DOI: 10.19082/6829 PMid: 29997768 PMCid: PMC6033126

5) CDAC. e-sushrut. Center for Development of Advanced Computing. Available from: https://www.cdac.in/index.aspx?id=hi his sushrut

6) Haryana State Health Resource Center. Available from: http://hshrc.gov.in/e-Upchaar.html

7) Kharbanda O, Priya H, Bhadauria U, Khurana C, Das D. mOral health in India: Current scenario and future perspectives. Contemporary Clinical Dentistry. 2019;10:586. doi: 10.4103/ccd.ccd_824_18

8) Haryana Government, India .e-Gazette. Available from: https://haryana.gov.in/e-gazette

9) Lucas H. Information and communications technology for future health systems in developing countries. Soc Sci Med. 2008;66(10):2122-32. DOI: 10.1016/j.socscimed.2008.01.033 PMid: 18343005

10) Moidu K, Singh AK, Boström K, Wigertz O, Trell E, Kjessler B. MCHS: an application software for family welfare programmes. Med Inform (Lond). 1992;17(4):279-91. DOI: 10.3109/14639239209079810 PMid: 1305704

11) Braa J, Macome E, Mavimbe JC, Nhampossa JL, da Costa JL, José B, et al. A Study of the Actual and Potential Usage of Information and Communication Technology at District and Provincial Levels in Mozambique with a Focus on the Health Sector. Electron J Inf Syst Dev Ctries. 2001;5(1):1-29. DOI: 10.1002/j.1681-4835.2001.tb00031.x

12) Saluvan M, Ozonoff A. Functionality of hospital information systems: Results from a survey of quality directors at Turkish hospitals. BMC Med Inform Decis Mak. 2018;18(1). DOI: 10.1186/s12911-018-05812 PMid: 29329532 PMCid: PMC5767047

13) Krishnan A, Nongkynrih B, Yadav K, Singh S, Gupta V. Evaluation of computerized health management information system for primary health care in rural India. BMC Health Serv Res. 2010; 10. DOI: 10.1186/1472-6963-10-310 PMid: 21078203 PMCid: PMC2996385

14) Downey L, Rao N, Guinness L, Asaria M, Prinja S, Sinha A, et al. Identification of publicly available data sources to inform the conduct of Health Technology Assessment in India. F1000Research. 2018; 7:245. DOI: 10.12688/f1000research.14041.2 PMid: 29770210 PMCid: PMC5930391

15) Kalankesh L, Pourasghar F, Jafarabadi M, Khanehdan N. Depiction of Trends in Administrative Healthcare Data from Hospital Information System. Mater Socio Medica. 2015;27(3):211. DOI: 10.5455/msm.2015.27.211-214 PMid: 26236170 PMCid: PMC4499278

16) Arora $D$, Mehta $Y$. Use of picture archiving and communication system for imaging of radiological films in cardiac surgical intensive care unit. J Anaesthesiol Clin Pharmacol. 2014 Jul; 30(3):447-8. DOI: 10.4103/0970-9185.137306 PMid: 25190974 PMCid: PMC4152706 